\title{
AN OPTIMIZED SHAPE CAVITY FOR TESLA: CONCEPT AND FABRICATION ${ }^{\#}$
}

\author{
V. Shemelin*, R. L. Geng, J. Kirchgessner, H. Padamsee, J. Sears \\ Laboratory for Elementary-Particle Physics, Cornell University, Ithaca, NY 14853
}

\section{Abstract}

The accelerating gradient performance of superconducting niobium cavities is rapidly progressing as a result of reduced field emission due to improvement in surface preparation techniques. Beyond the field emission limitation, there exists a fundamental limit imposed by the critical magnetic field of niobium. One way to tackle this limit is to reduce the ratio of the peak magnetic field to the accelerating gradient so that a higher accelerating gradient is possible while the cavity is still superconducting. New cavity shapes of reentrant type have been proposed and optimized [1]. A single cell $1300 \mathrm{MHz}$ cavity of this new class of shapes has been fabricated. Because of the reentrant geometry, the fabrication and surface cleaning of the cavity becomes challenging. In this paper, we present some calculation prerequisites and the fabrication and preparation results for this new cavity.

\section{INTRODUCTION}

In deciding on a cell shape of a SC accelerating section, it is necessary to ensure both electric and magnetic strength. For comparison of different shapes one can use the ratios of the peak electric and magnetic field strength on the cell surface to the acceleration rate achievable in the given cell:

$$
\frac{E_{p k}}{E_{a c c}}=\frac{E_{p k}}{\Delta W / L}=\frac{E_{p k}}{2 \Delta W / \lambda}, \frac{H_{p k}}{E_{a c c}}=\frac{H_{p k}}{2 \Delta W / \lambda} .
$$

Here $\Delta W$ is the energy gain (in volts) obtained at the cell length $L$ equal to half wavelength ( $\pi$-mode). For the TESLA accelerating cavity these values are [2]:

$$
E_{p k} / E_{a c c}=2.0, H_{p k} / E_{a c c}=42 \mathrm{Oe} /(\mathrm{MV} / \mathrm{m}) \text {. }
$$

We will compare values of calculated fields with these values and introduce for this purpose the normalized peak electric and magnetic fields:

$$
e=\frac{E_{p k}}{2 E_{a c c}}, h=\frac{H_{p k}}{42 E_{a c c}} .
$$

For the regular TESLA cells [2]

$$
e=1, \quad h=1 \text {. }
$$

We believe that for superconducting cavities it is more important to reduce $H_{p k}$ on the surface, even if we increase $E_{p k}$. This is because the critical magnetic field is a hard limit at which superconductivity fails and the cavity quenches; whereas $E_{p k}$ is a soft limit: field emission can be decreased by maintaining better cleanliness and by high power processing.

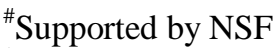

*vs65@cornell.edu
}

\section{THE CODE AND GEOMETRY FOR CALCULATIONS}

We used for optimization the SLANS code [3]. This code has better accuracy in comparison with earlier URMEL code used for calculation of TESLA cavities. With SLANS we can expect accuracy better than $0.1 \%$.

The profile line of the original TESLA cell is constructed as two arcs: elliptic and circular, and a segment of a conjugated straight line between them (the dashed line in Fig. 1). It is felt that more intricate line could give better values of $e$ and $h$.

Between other approaches we examined [1], one was done with use of two elliptic arcs. The problem of a cavity electric strength led to the iris edge in a shape of an ellipse long ago [4]. This type of cavity has several features important for superconducting $\mathrm{Nb}$ cavities [5]. Let us apply an ellipse to the inductive part of the cell because now we have a problem of magnetic strength.

After some optimization of the original geometry, the length of the straight interval conjugated to both ellipses appeared to be zero. So, we could describe the shape of the regular cell as two conjugated elliptic arcs. The aperture was taken as in the TESLA cell, $R_{a}=35 \mathrm{~mm}$.

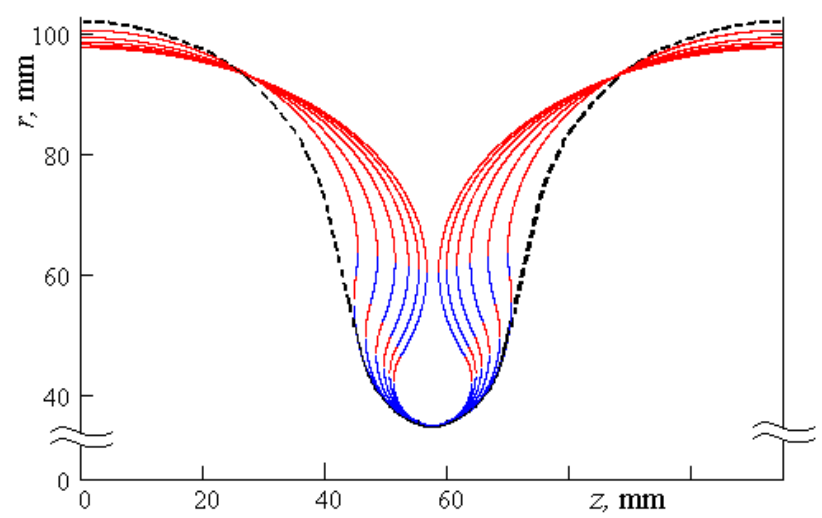

Fig. 1. Optimization of the TESLA regular cell shape.

Dashed line - the present shape, solids - optimization with 2 elliptic arcs, $\delta e=0,10, \ldots, 50 \%$.

\section{RESULTS OF CALCULATIONS}

The process of optimization consists in searching a cell shape with a minimal value of the peak normalized surface field $e$ in this cell. The result of optimization should be a function $h(e)$, which should be a monotonous function, and from physical reasons it should be decreasing.

We turn to maximal electric strength as a special case: maximal electric strength is achieved with the shape corresponding to the leftmost point on the curve $h(e)$. 
The results obtained by optimization can be used at any operation frequency because the values of $e$ and $h$ depend on the shape, not on the dimensions of the cavity. On the other hand, the value of the critical field $H$ depends on the frequency and the choice of the working point on the curve of Fig. 2 can be different for different frequencies.

$h$ goes down for lower ratios of the beam pipe radius to the wavelength ( $R_{a} / \lambda$, Fig. 2 ). However, it is preferable to keep the same $R_{a}$ to preserve low wakefields.

The data presented on the Fig. 2 correspond to the regular cell of the structure. The end cells have somewhat different dimensions and their optimization should be discussed separately.

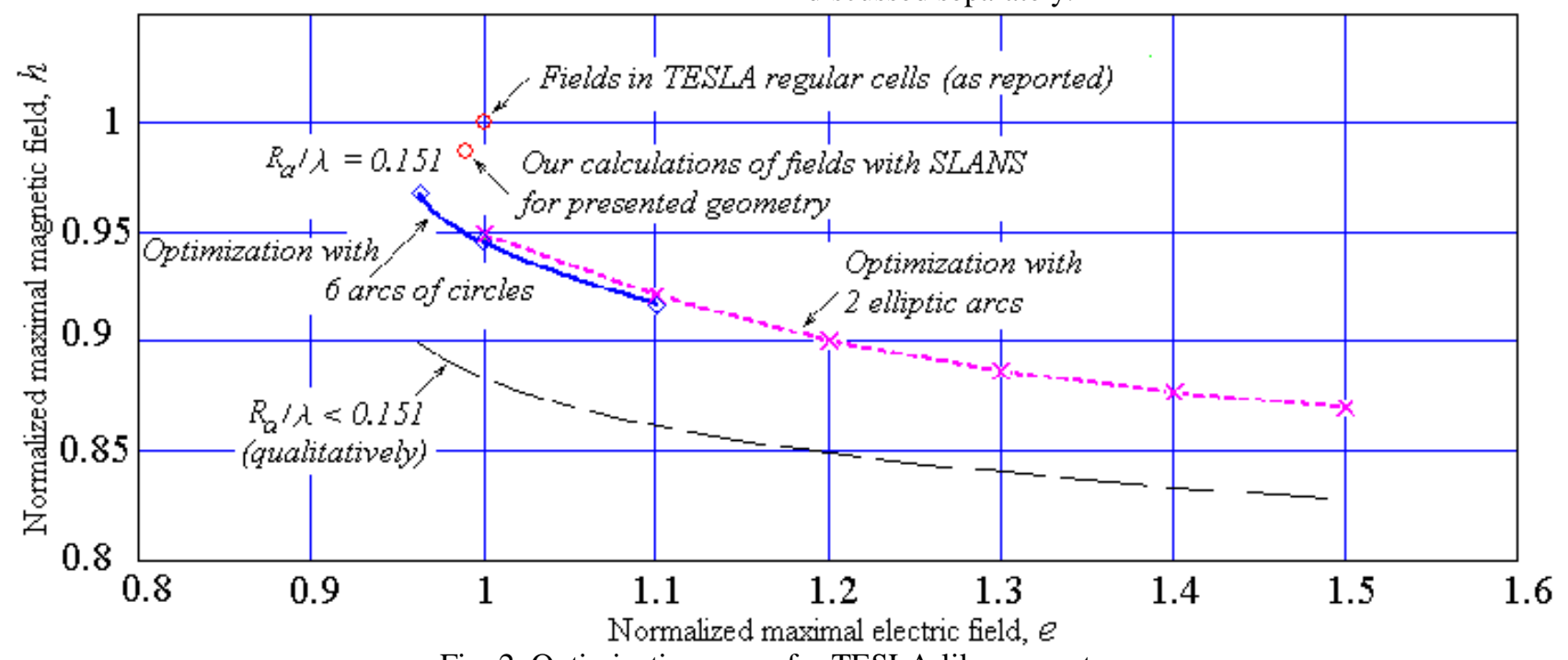

Fig. 2. Optimization curve for TESLA-like geometry.

Our calculations showed that for the TESLA regular cell geometry [2] maximal fields differ slightly from the values of fields presented also there: the electric field is $1.2 \%$ below and magnetic field is $1.3 \%$ below for the regular cells (see Fig. 2). The coupling coefficient $k$ is obtained nearly the same: $1.87 \%$.

For comparison the results are summarized in the Table. Values of cell-to-cell coupling and iris thickness are also presented. This thickness $(d)$ becomes small for the biggest $\delta e$ that can be a technological problem.

Distribution of the cell area over the electric field is presented in Fig 3 for the original TESLA shape and for the shape with $10 \%$ less magnetic field.

The parts of the curves corresponding to the field within $90 \ldots 100 \%$ of the maximal value are shown. The area under each curve corresponds to the portion of the whole cell surface having the given value of field. One can see, for example, that $1.6 \%$ of surface has electric field within $99.5 \ldots 100 \%$ of the maximal value in case of the optimal cell. The original cell has $2.8 \%$ of surface within the same range of the relative value of field. However, the maximal field is 1.2 higher in the case of optimized field. The consequence of this is a higher field emission current in spite of less area. For example, for the field enhancement factor $\beta=200$ and accelerating field $E_{a c c}=35 \mathrm{MV} / \mathrm{m}$ the total field emission current will be 2.7 times higher for the optimized cell. This is a payment for a lower magnetic field.

With the simulation code MultiPac [6], it was shown that multipacting characteristics of the new cavity shape are not worse than of the original TESLA shape.
Table. $\delta e, \delta h$ - change of normalized electric and magnetic fields by optimization with two elliptic arcs, $k-$ coupling coefficient, $d$ - minimal distance between the walls of cells.

\begin{tabular}{|c|c|c|c|}
\hline$\delta e, \%$ & $\delta h, \%$ & $k, \%$ & $d, \mathrm{~mm}$ \\
\hline 0 & -5.07 & 1.90 & 24.80 \\
\hline+10 & -7.92 & 2.10 & 18.30 \\
\hline+20 & -10.00 & 2.38 & 12.52 \\
\hline+30 & -11.36 & 2.64 & 8.14 \\
\hline+40 & -12.30 & 2.88 & 4.74 \\
\hline+50 & -12.99 & 3.06 & 2.18 \\
\hline
\end{tabular}

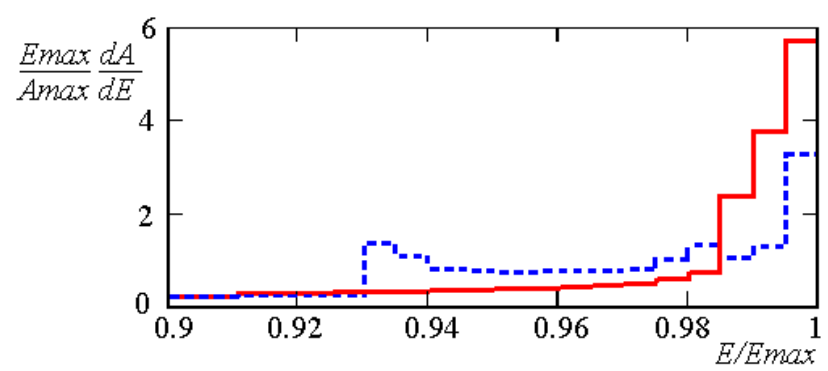

Fig. 3. Distribution of the cell surface area over the electric field. Solid line is for the original TESLA shape, dashed line - for the optimized cell with $10 \%$ lower magnetic and $20 \%$ higher electric field. $A_{\max }$ is the total surface area of the cell.

\section{FABRICATION}

Reentrant cups were successfully formed by deep drawing $3 \mathrm{~mm}$ thick RRR300 sheet niobium. It was critical to hold down the niobium blank sheet to the 
female die with a right torque. A center hole (an inch in diameter) on the blank was found beneficial to achieve better material flow in the critical reentrant section. No intermediate annealing was done to the cups. Very good contour of the reentrant section was achieved by using the coining technique, although the reentrant section was not pushed deep enough after the first stamping step. In order to reduce the chances of cavity quench at high gradients, cups were purified with yttrium [7] to drive out oxygen and improve niobium thermal conductivity. Heat treatment was done at $1200^{\circ} \mathrm{C}$ for 4 hours with distributed yttrium foils in the vicinity of niobium surface. The RRR value was boosted to 500 after this purification. The cups were trimmed with standard milling process. Fig. 4 shows a pair of the trimmed niobium cups to be welded to beam tubes. We plan to electropolish the half-cell before the final equatorial weld. This desire has driven us to switch from our traditional "step weld" of 1/16" material to "butt weld" of thicker, 0.11", material. A new set of EBW parameters has been successfully developed and a very high quality weld with a negligible underbead was obtained. This is achieved by opening a small compensating groove on the inner surface at the equator, which holds the molten niobium and maintains its tensioned surface at the right place. Beam tubes are welded to the half-cells first. The welded parts would then go through heavy electropolish for a surface removal of more than 120 microns. This "half-cell electropolish" is the same as what we have done to some $1500 \mathrm{MHz}$ cavities and half-cells [8]. The final equatorial weld of electropolished half-cells is done from outside and at full penetration with a niobium rod running across irises, which serves to intercept niobium vapor and spatter and prevents contamination to polished surface. The cavity would receive a light $\mathrm{BCP}$, for a surface removal of about 10 microns, before high-pressure water rinsing, following which will be RF tests.

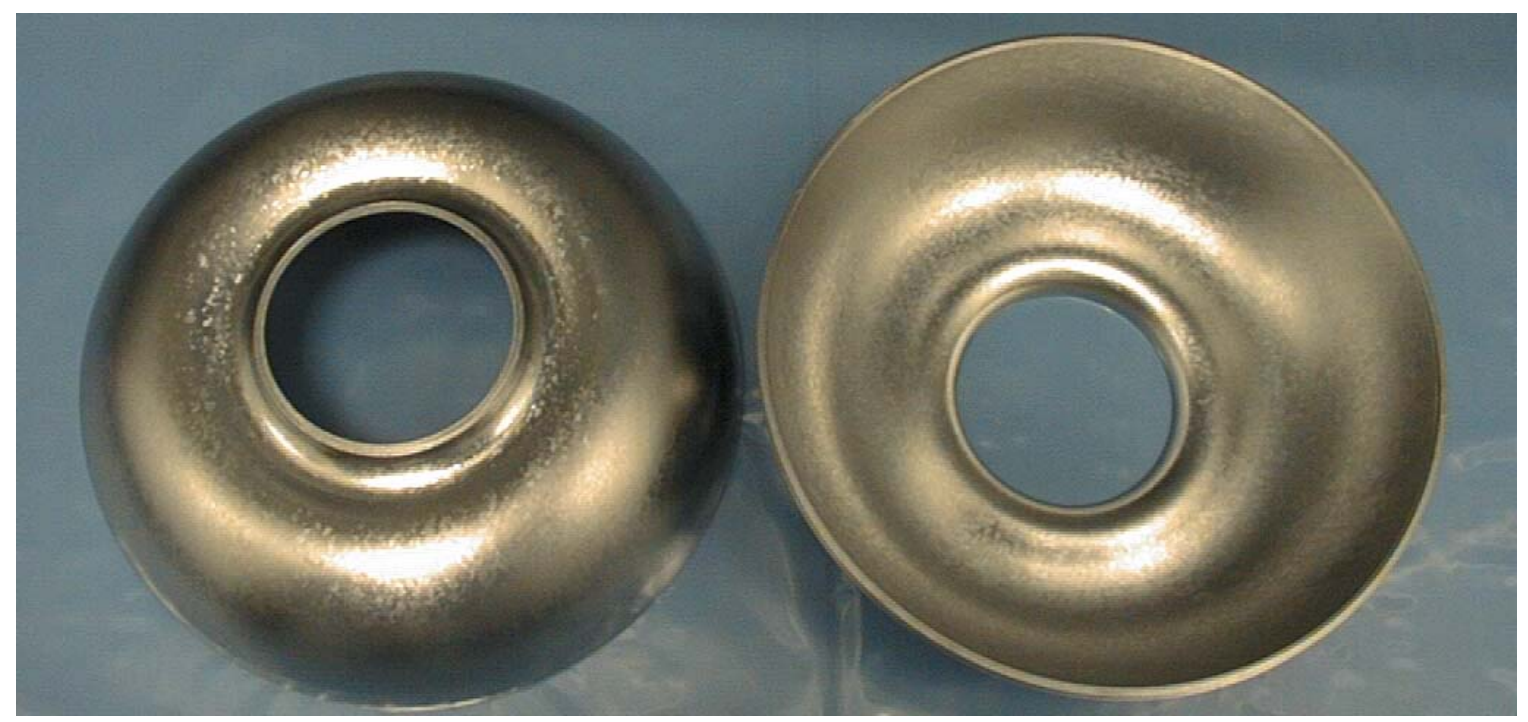

Fig. 4. $1300 \mathrm{MHz}$ reentrant cavity cups. Left cup: outside surface view, right cup: inside surface view.

\section{CONCLUSIONS}

The presented results can be used for an increase of accelerating rate of the TESLA structure where the hard limit for this increase is the surface magnetic field. One can, for example, sacrifice $20 \%$ of electric field to gain $10 \%$ in magnetic field.

Calculations show that the new shape should be free of multipacting.

The change of the shape leads to some technological complications. Reentrant cups were successfully formed, heat-treated and prepared to electropolishing and welding.

\section{REFERENCES}

[1] V. Shemelin, H. Padamsee, R.L. Geng. Optimal cells for TESLA accelerating structure. Nucl. Instr. Methods Phys. Res.-A, 496, pp. 1-7, 2003.

[2] TESLA Test Facility Linac - Design Report. Editor D.A. Edwards. DESY Print, March 1995, TESLA 95-01.
[3] D.G. Myakishev, V.P. Yakovlev. The new possibilities of SuperLANS code for evaluation of axisymmetric cavities. 1995 PAC and Int. Conf. on High-Energy Accel. May 1-5, 1995. Texas. Pp. 2348-2350.

[4] M.M. Karliner, O.A. Nezhevenko, B.M. Fomel, V.P.Yakovlev. On the problem of comparison of accelerating structures operated by stored energy. (In Russian). Preprint of INP 86-146, Novosibirsk, 1986.

[5] P. Kneisel, R. Vincon and J. Halbritter, First results on elliptically shaped cavities. Nucl. Instr. Methods Phys. Res., 188, p.669-670, 1981.

[6] P. Ylä-Oijala et al. MultiPac 2.1. Rolf Nevanlinna Institute, Helsinki, 2001.

[7] H. Padamsee, IEEE Trans. Magn., 21, 1007 (1985).

[8] R.L. Geng et al., A $1500 \mathrm{MHz}$ niobium cavity made of electropolished half-cells. This Conference, report TPAB050. 